\section{Glioma difuso de línea media H3K27M positivo en adulto. Caso clínico}

\author{
PATRICIA ARANEDA ${ }^{1}$, EMI SUJIMA ${ }^{1,2}$, \\ PATRICIO PAREDES-MANZO ${ }^{3}$, RODRIGO VALLEJO ${ }^{4}$, \\ FELIPE VALDIVIA ${ }^{4}$, MARIANA SINNING ${ }^{1,2}$, \\ MARCELA GALLEGOS ${ }^{5}$, GREGORY FULLER ${ }^{6}$
}

\section{H3K27 positive diffuse midline glioma. Report of one case}

The 2016 WHO Classification of Tumours of the Central Nervous System incorporates a new diagnostic entity: the mutant diffuse midline glioma H3K27, a tumor with a characteristic location and special molecular biology. We report the case of a 51-year-old male patient with progressive diplopia. The imaging study showed a mesencephalic tumor; the stereotacic biopsy disclosed an Anaplastic Astrocytoma Isocitrate dehydrogenase (IDH) wild type. The molecular study concludes H3K27 mutation. The patient was treated with radiotherapy with concurrent and adjuvant chemotherapy (temozolomide) with partial recovery of the diplopia.

(Rev Med Chile 2019; 147: 1487-1490)

Key words: Astrocytoma; Glioma; Histones.
'Departamento Neurología-

Psiquiatría, Clínica Alemana.

Santiago, Chile.

${ }^{2}$ Departamento de Oncología,

Clínica Alemana. Santiago, Chile.

${ }^{3}$ Departamento de Imagenología,

Clínica Alemana. Santiago, Chile.

${ }^{4}$ Departamento Cirugía, Clínica

Alemana. Santiago, Chile.

${ }^{5}$ Departamento Laboratorio

Clínico, Banco de Sangre y

Anatomía Patológica, Clínica

Alemana. Santiago, Chile.

${ }^{6}$ Department of Pathology, MD

Anderson Cancer Center. Texas,

Estados Unidos.

Trabajo no recibió financiamiento. Los autores declaran no tener conflictos de interés.

Recibido el 25 de abril de 2019, aceptado el 9 de diciembre de 2019.

Correspondencia a:

Mariana Sinning,

Departamento Neurología-

Psiquiatría, Clínica Alemana

Santiago, Chile.

msinning@alemana.cl
L a clasificación de tumores primarios de SNC de la OMS publicada el 2016 ${ }^{1}$, introduce la necesidad de contar con marcadores moleculares que permiten agrupar entidades con biología y comportamiento clínico similar². Como entidad nueva se incorpora el glioma difuso de línea media H3K27 mutado, que se presenta en una localización característica (troncoencéfalo, tálamo y médula) y con edad característica (niños y adultos jóvenes). En este reporte presentamos el caso de un paciente de 51 años, portador de un glioma talámico: astrocitoma anaplásico H3K27m. Hasta donde sabemos, este corresponde al primer caso reportado en Latinoamérica en un adulto diagnosticado con estudio molecular.

\section{Caso clínico}

Paciente hombre de 51 años, con antecedentes de hipertensión arterial y diabetes mellitus, que presenta 4 meses de diplopia horizontal progre- siva. Asociado a cefalea holocránea, opresiva, de intensidad fluctuante y en las semanas previas al diagnóstico, apraxia de la marcha. Sin antecedentes familiares. Al examen neurológico de ingreso destaca el compromiso de un VI nervio craneal bilateral, mayor a derecha y apraxia de la marcha. El examen sistémico sin hallazgos. Se realiza RM de cerebro (Figura 1), examen que pesquisa hidrocefalia no comunicante y proceso expansivo mesencefálico de carácter tumoral. Se interviene en un primer tiempo para colocación de una derivativa ventrículo peritoneal con resolución del cuadro de hidrocefalia. Se prefiere esta técnica dada la localización intra-axial por debajo del plano ependimario de la lesión cuyo centro se ubicaba hacia mesencéfalo. Se realiza luego una biopsia estereotáxica con sistema de neuronavegación, obteniendo una muestra representativa de la lesión. Con buena respuesta clínica con desaparición de sus síntomas de hipertensión endocraneana, sin complicaciones locales o empeoramiento en su situación neu- 
rológica, pudiendo manejarse sin corticoides y controles de imágenes sin hidrocefalia. El estudio histológico de la muestra confirma el diagnóstico de astrocitoma anaplásico OMS Grado III IDH 1 y 2 nativo y MGMT no metilado. Dada la localización diencefálica, específicamente por tratarse de glioma talámico, (localización característica de glioma con mutación H3K27), con IDH nativo, se decide complementar el estudio con análisis molecular en MD Anderson Cancer Center, demostrando expresión de proteína mutante H3K27 en células gliomatosas y pérdida de K27me3 por inmunohistoquímica, PDGFRA positivo, p53 negativo, ATRX presente (Figura 2). Tras análisis del caso en comité de neuro-oncología, se inicia tratamiento según protocolo de Stupp: Radioquimioterapia concomitante y quimioterapia adyuvante. Al término de la radioquimioterapia el paciente ha presentado mejora parcial de su diplopia, actualmente en adyuvancia con temozolomida. Se realizó proceso de consentimiento informado para el reporte del caso actual.
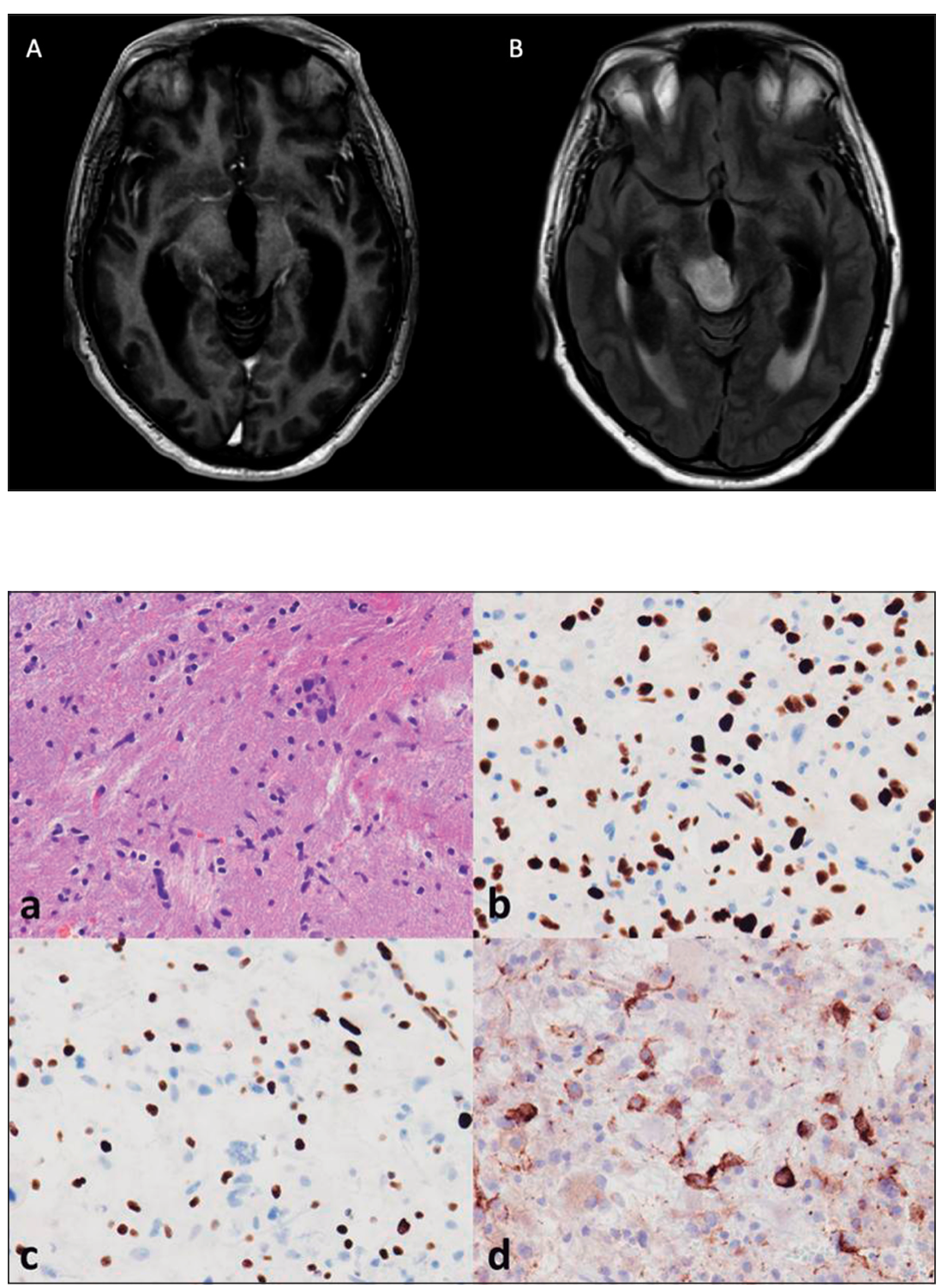

Figura 1. Resonancia magnética de cerebro. A: Secuencia T1 con medio de contraste; B: Secuencia FLAIR.
Figura 2: Histología. A: tinción hematoxilina eosina. B: inmunohistoquímica $\mathrm{H} 3 \mathrm{~K} 27 \mathrm{~m}$ que evidencia tinción nuclear positiva. C: inmunohistoquímica K27me3, pérdida de expresión en células tumorales. D: inmunohistoquímica para PDGFRA. 


\section{Discusión}

El 2016 se publicó la última clasificación de la OMS de tumores primarios de SNC, logrando un avance práctico y conceptual en relación a las clasificaciones previas ${ }^{1}$. Combinando fenotipo con genotipo de los tumores, logra agrupar entidades diagnósticas con un comportamiento biológico más homogéneo ${ }^{3}$. Dentro de dicha clasificación se incorpora una nueva entidad: el glioma difuso de línea media con mutaciones de sustitución de lisina a metionina en el codón $27(\mathrm{~K} 27 \mathrm{M})^{4}$ en la histona H3. Esta mutación se describe por primera vez en gliomas difusos pontinos ${ }^{5}$ y luego en gliomas de línea media. Si bien el mecanismo oncogénico se encuentra aún en estudio, la sustitución de lisina por metionina, disminuye la trimetilación de $\mathrm{K} 27 \mathrm{~m}$, impidiendo la represión de áreas del genoma. Descubrimientos como éste llevan al concepto de subgrupos de gliomas de alto grado, en donde la célula progenitora sufre insultos específicos desarrollando entidades con un comportamiento biológico similar, siendo la mutación $\mathrm{H} 3.3 \mathrm{~K} 27 \mathrm{M}$ característica en tumores de línea media y H3.1K27M en tumores de puente ${ }^{6}$. Los análisis en estos pacientes han revelado $\mathrm{H} 3 \mathrm{~K} 27$ es mutuamente excluyente con IDH 1 y amplificación de EGFR y cooperantes, en especial con sobreexpresión de $\mathrm{p} 53^{7}$ y pérdida de expresión nuclear del ATRX ${ }^{4}$ : hasta en $50 \%$ de los casos se encuentra alteraciones de p53, en 30\% de PDGFRA y en $15 \%$ de ATRX.

La mutación H3K27M ha sido descrita predominantemente en niños ${ }^{8} y$ en adultos, suelen ser adultos jóvenes ${ }^{5}$.

El contar con el diagnóstico molecular de H3K27 tiene importancia a nivel diagnóstico, en ciertos casos el aspecto morfológico no es sugerente de un glioma de alto grado9. También tiene importancia pronóstica, pues se asocia a peor pronóstico, con una sobrevida menor a $10 \%$ a los 2 años $^{10}$. La mediana de sobrevida se describe entre distintas series entre 9-15 meses $^{4,9}$.

Onc 201 es una molécula pequeña, el primer miembro de una clase de compuestos de imipridona, es un inductor de la transcripción del gen TRAIL independiente de p53, con propiedades anti proliferativas con una alta selectividad en la inducción de apoptosis en células malignas ${ }^{11}$, además la mutación H3K27M parece sensibilizar a las células tumorales a la acción de este fármaco. En un estudio fase II publicado recientemente en pacientes con glioblastoma IDH nativo recurrente se observó en un paciente con enfermedad multifocal portador de la mutación $\mathrm{H} 2 \mathrm{~K} 27$, una reducción tumoral de $85 \%$ en una lesión y $76 \%$ de la lesión secundaria ${ }^{12}$. Actualmente se encuentra en reclutamiento 2 estudios fase II para el tratamiento con ONC201 de gliomas difusos H3k27M en adultos con enfermedad recurrente (NCT03295396/ NCT02525692), además de extensa investigación preclínica es búsqueda de nuevas moléculas ${ }^{13}$.

Actualmente en nuestro país no se realiza el estudio de la mutación de histona H3K27M, sería de importancia su incorporación como marcador con utilidad diagnóstica, pronóstica y esperamos en un futuro próximo, predictor de respuesta a tratamiento.

\section{Referencias}

1. Louis DN, Perry A, Reifenberger G, Deimling A Von, Figarella D, Webster B, et al. The 2016 World Health Organization Classification of Tumors of the Central Nervous System: a summary. Acta Neuropathol 2016; 131 (6): 803-20.

2. Sinning M. Clasificación de los tumores cerebrales. Rev Med Clin Condes 2017; 28 (3): 339-42.

3. Porkholm M, Hernesniemi J, Oinas M, Raunio A, Valanne L, Vainionpää R, et al. Molecular alterations in pediatric brainstem gliomas. Pediatr Blood Cancer 2018; 65 (1): e26751.

4. Solomon D, Wood M, Tihan T, Bollen A, Gupta N, Phillips J. Diffuse Midline Gliomas with Histone H3-K27M Mutation: A Series of 47 Cases Assessing the Spectrum of Morphologic Variation and Associated Genetic Alterations. Brain Pathol 2018; 26 (5): 569-80.

5. Kleinschmidt-DeMasters BK, Levy JMM. H3 K27M-mutant gliomas in adults vs children share similar histological features and adverse prognosis. Clin Neuropathol 2018; 37 (3): 53-63.

6. Jones C, Karajannis MA, Jones DTW, Kieran MW, Monje M, Baker SJ, et al. Pediatric high-grade glioma: biologically and clinically in need of new thinking. Neuro-Oncology 2017; 19 (2): 153-61.

7. Nichol JN, Ezponda T, Licht JD, Centre C, Hospital JG, Medicine E. H3K27 Methylation: A Focal Point of Epigenetic Deregulation in Cancer. Adv Cancer Res 2017; 131: 1-33.

8. Bailey CP, Figueroa M, Mohiuddin S, Zaky W. Cutting Edge Therapeutic Insights Derived from Molecular 
Biology of Pediatric High-Grade Glioma and Diffuse Intrinsic Pontine Glioma (DIPG). Bioengineering 2017; 19 (2): 153-61.

9. Meyronet D, Esteban-Mader M, Bonnet C, Joly M, Uro-Coste E, Amiel-Benouaich A, et al. Characteristics of H3 K27M-mutant gliomas in adults. Neuro-Oncology 2017; 19: 1127-34.

10. Jansen MH, Zanten SEV Van, Aliaga ES, Heymans MW, Warmuth- M, Hargrave D, et al. Survival prediction model of children with diffuse intrinsic pontine glioma based on clinical and radiological criteria. Neuro-Oncology 2015; 17: 160-6.
11. Allen JE, Kline CLB, Prabhu VV, Wagner J, Ishizawa J, Madhukar N, et al. Discovery and clinical introduction of first-in-class imipridone. Oncotarget 2016; 7 (45): 74380-92.

12. Arrillaga-Romany I, Chi AS, Allen JE, Oster W, Wen PY, Batchelor TT. A phase 2 study of the first imipridone ONC201, a selective DRD2 antagonist for oncology, administered every three weeks in recurrent glioblastoma. Oncotarget 2017; 8 (45): 79298-304.

13. Long W, Yi Y, Chen S, Cao Q, Zhao W, Liu Q. Potential New Therapies for Pediatric Diffuse Intrinsic Pontine Glioma. Front Pharmacol 2017; 8: 1-13.

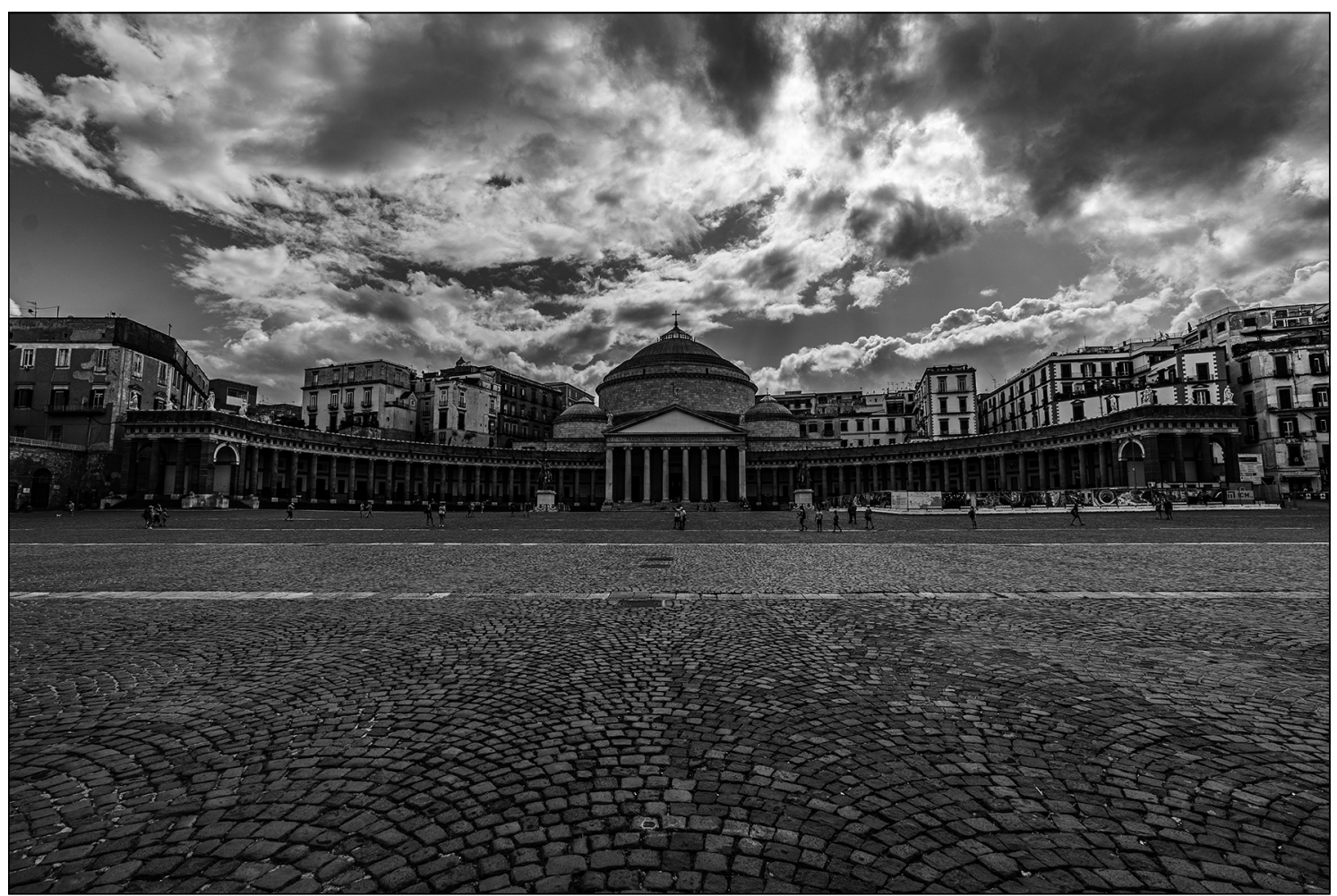

Plaza del Plebiscito, Nápoles. Dr. Francisco Cordero Anfossi 